\title{
Interaction of Satiety and Reward Response to Food Stimulation
}

\author{
G. Andrew James, $\mathrm{PhD}$ \\ Mark S. Gold, MD \\ Yijun Liu, PhD
}

\begin{abstract}
SUMMARY. Obesity is among the most pressing health issues affecting developed countries. The etiology of obesity remains unclear despite its associated health risks and problems. We propose a framework for obesity modeled upon overeating as a substance dependence disorder arising from a combination of abnormal cognitive and neuroendocrine processes. While significant work has investigated the body's regulation of satiety signals, fewer studies have focused on the mechanisms by which these two seemingly disparate (cognitive and neuroendocrine) systems interact. Although emotional states have been shown to mediate reward processing, the implications for hunger mediating reward have not previously been addressed. We review the interaction between cen-
\end{abstract}

G. Andrew James, Mark S. Gold, and Yijun Liu are affiliated with the University of Florida College of Medicine, Departments of Psychiatry and Neuroscience, and McKnight Brain Institute, Gainesville, FL.

Address correspondence to: Yijun Liu, PhD, The University of Florida McKnight Brain Institute, Department of Psychiatry, 100 South Newell Drive, L4-100, Gainesville, FL 32610-0256.

The authors are grateful to Dr. A. G. He for assistance in MRI data acquisition. This work was supported in part by grant from National Institutes of Health (NS45518 and DA16221).

[Haworth co-indexing entry note]: "Interaction of Satiety and Reward Response to Food Stimulation." James, G. Andrew, Mark S. Gold, and Yijun Liu. Co-published simultaneously in Journal of Addictive Diseases (The Haworth Medical Press, an imprint of The Haworth Press, Inc.) Vol. 23, No. 3, 2004, pp. 23-37; and: Eating Disorders, Overeating, and Pathological Attachment to Food: Independent or Addictive Disorders? (ed: Mark S. Gold) The Haworth Medical Press, an imprint of The Haworth Press, Inc., 2004, pp. 23-37. Single or multiple copies of this article are available for a fee from The Haworth Document Delivery Service [1-800-HAWORTH, 9:00 a.m. - 5:00 p.m. (EST). E-mail address: docdelivery@ haworthpress.com].

http://www.haworthpress.com/web/JAD

(C) 2004 by The Haworth Press, Inc. All rights reserved.

Digital Object Identifer: 10.1300/J069v23n03_03 
tral satiety signals and reward responses to food stimuli and discuss the implications of this research for understanding the causes of obesity. [Article copies available for a fee from The Haworth Document Delivery Service: 1-800-HAWORTH. E-mail address: <docdelivery@ haworthpress.com>Website: 〈http://www.HaworthPress.com> () 2004 by The Haworth Press, Inc. All rights reserved.]

KEYWORDS. Neuroimaging, fMRI, obesity, overeating

\section{INTRODUCTION}

Obesity is a serious health condition reaching pandemic proportions. Recent surveys indicate that, in the United States, one-third of men and women aged 20 years or older are overweight [body mass index (BMI) $>25.0] .{ }^{1}$ The percentage of clinically obese (BMI > 30.0) individuals in the US has nearly doubled in the past two decades. ${ }^{2}$ Health problems linked to obesity are numerous and include stroke, heart disease, noninsulin dependent diabetes mellitus, osteoarthritis, and increased risk for developing cancer. ${ }^{3,4}$ The number of deaths relating to obesity rivals those attributed to alcohol- and nicotine-use. ${ }^{5}$ But while researchers agree that obesity is a disease warranting increased public awareness, its relationship to depression, personality disorders and addiction are not as strongly emphasized. The similarities between overeating and substance use disorders have been established ${ }^{6,7}$ as have the co-morbid disorders most commonly associated with these illnesses. Recent functional brain imaging studies have suggested that obesity and the inability to control eating behaviors produce changes in neural activity patterns similar to those produced by substance use. ${ }^{8,9}$ Given these similarities, newly discovered physiological messengers that modulate eating behavior (i.e., leptin and ghrelin) may mediate not only obesity but also alcoholism and other drug dependencies.

\section{THEORETICAL CAUSES OF OBESITY}

Numerous theories attempt to explain the causes of obesity. A popular biologic theory is that obesity develops from abnormal neuroendocrine processes involved in the control of eating behavior and energy homeostasis. Most of these theories focus upon the hypothalamus, a principal component of the central nervous system for maintain- 
ing energy homeostasis. ${ }^{10,11}$ An alteration in hypothalamic response to anorexigenic or orexigenic signals could result in a delayed central sensation of satiety. The consequent feeding behavior would gradually lead to obesity.

Conversely, the prototypical cognitive approach cites the social implications of food as reward (e.g., having to clean one's plate for dessert) and focuses upon our behavioral responses to food rewards. ${ }^{12}$ While these disparate approaches may initially seem irreconcilable, the regulation of hunger and satiety most likely stems from interaction between endocrine and cognitive processes. ${ }^{13}$ The purpose of this review is to elaborate upon psychobiological processes mediating hunger and satiety.

\section{Overeating as a Substance Dependence Disorder}

Constructing a model for overeating as an addiction is inherently difficult due to the ambiguous psychological and psychiatric definitions of addiction. We instead model overeating as a substance dependence disorder. The comparison is imperfect since the DSM-IV does not recognize food as a substance of abuse. Furthermore, we are all physiologically dependent upon food for survival, so one could facetiously argue that everyone suffers from tolerance and withdrawal to food (two of three specifiers necessary for a diagnosis of substance dependence). The remaining specifiers for substance dependence (e.g., greater consumption of the substance than planned, failed attempts to cut back on consumption, etc.) reflect the difficulties many obese individuals experience when attempting to reduce food intake. When the criteria for physiological dependence are disregarded, food adequately fits the model for substance dependence.

We contend that obesity may be considered a byproduct of substance dependence with food as the substance in question. Within this theoretical framework, cognitive-behavioral therapies typically reserved for substance dependence therapies may be applicable toward the treatment of obesity. ${ }^{14}$ However, treatment is beyond the scope of this review; we instead wish to review prominent functional neuroimaging research on the reward system and their common implications for substance dependence and obesity. We will also review a novel functional MRI (fMRI) method for studying the neuro-hormonal mediation of hunger and satiety based on our previous work, ${ }^{15,16}$ focusing on the hypothalamus and its associated signaling pathways in regulating eating behavior and body-weight. Our ultimate goal is to provide a feasible model for 
the interaction of the nervous and endocrine systems in regulating both substance use and eating behaviors.

\section{RECENT METHODOLOGY DEVELOPMENT AND FINDINGS}

\section{Functional Neuroanatomy of Reward}

Reward-system circuitry and the learning of reward contingencies have been topics of study for many years. Excellent reviews can be found concerning the role of neurotransmitters in establishing and mediating reward incidents ${ }^{17-19}$ as well as the neuroanatomy and connectivity of reward circuits. ${ }^{20-22}$ These reviews stress two main circuits for mediating reward behaviors: a fronto-amygdalar pathway reciprocally connecting the amygdala and prefrontal (orbito-, ventromedial-, and dorsolateral-) cortices and a limbic circuit integrating the amygdala with the hypothalamus and septal nuclei. A second limbic circuit, Papez circuit, integrates the hypothalamus with hippocampus and thalamus. The hypothalamus is at the junction between these two limbic circuits, with the hippocampal branch forming new memories while the amygdalar branch assigns value judgments to those memories.

The tight connectivity of the limbic circuits with phylogenically ancient structures such as the insular cortex suggests that the limbic system is mostly concerned with unconditionally rewarding stimuli such as food, water, and sex. ${ }^{23,24}$ In contrast, the fronto-amygdalar circuit may be more focused upon conditionally rewarding stimuli, such as money or abstract concepts. ${ }^{25}$ The distinction, if any, between the rewards these circuits mediate can best be teased apart with functional imaging. More than merely a means for corroborating anatomical findings, functional imaging techniques including positron emission tomography (PET) and fMRI allow the unique perspective of viewing brain circuitry in vivo. ${ }^{15,26}$ The inherently global nature of these techniques allows for the analysis of the spatial and temporal extent to which these anatomic reward circuits are activated. Individual differences in brain response to different rewarding stimuli can be assessed, as well as how these reward systems are perturbed by long-term drug abuse.

\section{Substance Dependence}

Nowhere is the synthesis of biological and physiological reward mechanisms more prominent than in substance dependence literature. 
Nicotine dependence, for example, can produce cue- and environmentdependent cravings so powerful that substance users will selectively pursue nicotine reinforcement over competing unconditional reinforcers. ${ }^{27,28}$ Substance dependence is particularly relevant to the discussion of reward since dependent users will continue to pursue the substance in question despite punishing factors inherent to substance use (i.e., unsanitary environments, detrimental health effects) and from external sources (i.e., disapproval of family and peers, legal and economic consequences). Long-term substance use results in physiological changes in the responsiveness of reward circuitry to the substance of use. ${ }^{29}$

Cocaine dependence exemplifies these changes in the neural systems mediating reward. Bonson et al. monitored the neural activity of people with cocaine dependencies as they were exposed to cocaine-related cues and neutral cues. ${ }^{30}$ The previous study on cocaine users demonstrated increased activation of the right dorsolateral prefrontal cortex, left lateral orbitofrontal cortex, and left ventrolateral amygdala in response to cocaine-related cues over neutral cues, with the activity of these regions positively correlating to the participants' self-reported degree of cocaine craving. Importantly, increased activity was not observed in areas not associated with reward, such as the paracentral cortex, posterior thalamus, globus pallidus and caudate nucleus, so the activation was specific to reward and not a global change in activity due to increased arousal. ${ }^{31}$ The observed patterns of reward circuitry activity generalize to other forms of addiction; for example, nicotine-deprived smokers demonstrate increased activation of both limbic circuits (the amygdala, hippocampus, ventral tegmental area, and thalamus) in response to smoking-cues over nonsmoking-cues. ${ }^{32}$ These previous studies emphasize that substances of abuse can alter both frontal and limbic responses.

It should come as no surprise that cues relating to substances of abuse activate the reward systems of individuals dependent upon those substances. But how does substance dependency affects the processing of non-substance rewards? Functional neuroimaging has shown activity of the limbic system and prefrontal cortices to vary with monetary gain or loss. ${ }^{33}$ While patients with nicotine-dependencies and healthy control populations had comparable limbic activity in response to monetary awards, dopaminergic areas such as the striatum were activated to monetary rewards in smokers and nonmonetary rewards in nonsmokers. ${ }^{34}$ One interpretation for these findings is that reward processing becomes anchored to the substance of dependence, and a given stimulus is rewarding only insofar as it can aid the subjects with addiction in obtain- 
ing the substance in question. However, it is possible that this decrease in activation is an inherent condition that predisposes a person toward obtaining concrete rewards such as drugs over abstract rewards. The causal nature of this relationship remains to be clarified with further research. Another interpretation of this study concerns the postulation proposed earlier that the fronto-amygdalar circuit deals with abstract, goal-oriented rewards whereas the limbic system focuses upon more basic rewards. Clearly, such an attribution is far more complex than previously stated if not patently false.

\section{Emotional Modulation of Reward}

The previously described studies pose the following question: does a pre-existing neurological condition predispose one toward substance dependence? While the answer to this question is beyond the scope of this review, aberrant responses to reinforcing stimuli have been observed in populations suffering from psychiatric disorders. It has recently been proposed that obsessive-compulsive disorder (OCD) is not merely an anxiety disorder but is also driven by disgust. ${ }^{35}$ Neuroimaging studies support this theory with the finding that OCD and control populations express similar patterns of brain activity in response to threatening visual-stimuli designed to induce fear and anxiety, whereas the activity patterns differ between the two groups for disgust-inducing stimuli. ${ }^{36}$ OCD patients viewing pictures of contaminated food had significant increases in insular activity (a region responsive to both gustatory stimuli and disgust) but significant decreases in medial prefrontal cortex activity relative to control populations. One can conclude that OCD patients found contamination-related stimuli more disgusting than did control subjects. These findings demonstrate an interaction between an individual's emotional state (in this case, disgust) and the valence of a stimulus. Although the causal nature of this relationship has not been formally established, emotion is most likely influencing reward processing in this instance since OCD is commonly considered an anxiety or affective disorder. Given the coterminous nature of the two limbic circuits, it is both feasible and probable that an affective disorder can alter the value judgments placed upon rewarding or punishing stimuli.

\section{The Biochemical and Physiological Processes of Satiety}

We have illustrated how psychiatric conditions such as addiction and OCD can influence the relative reward value of stimuli. The eating dis- 
order literature lends more credence to this theory. The endocrine response to fasting and the biochemical means by which the body mediates hunger and satiety is addressed (see Kalra \& Kalra: Overlapping and interactive pathways regulating appetite and craving, in this issue). But how do these neurochemical signaling pathways translate into behavior? What cognitive mechanisms influence reward processing so as to vary the reward value of food relative to other reinforcers as a function of the organism's satiety level? When and under what conditions does the interface between biochemical signaling and cognitive awareness occur?

Functional neuroimaging addresses these questions by monitoring the brain response to perturbations of the endocrine system. The fMRI technique of temporal clustering analysis (TCA) has been developed to identify when changes in brain activity occur throughout the course of a functional scan. ${ }^{15}$ TCA operates by searching the time course of a scan, second by second, for significant fluctuations in brain activity. Once it is determined when changes in brain activity occur, further analysis can be conducted to evaluate how and where brain response changes for these durations. TCA has been applied to assess changes in brain response to glucose administration in healthy fasting volunteers. Brain activity was shown to significantly change around 9 minutes following glucose ingestion in normal weight subjects (Figure 1). After isolating the duration of enhanced brain activity, statistical parametric mapping revealed significant decreases in hypothalamic activity, with this activity correlated with plasma insulin levels. ${ }^{9}, 15$ Interestingly, in obese subjects, such changes in fMRI measurement was significantly delayed and attenuated. ${ }^{16}$

Perhaps more intriguing is that plasma insulin levels did not correlate with the decreases in hypothalamic activity in obese fasting volunteers, ${ }^{9}$ while they did correlate in lean subjects. The lack of coordination between hypothalamic response and plasma insulin concentration conceivably translates into a delayed awareness of satiety. An inherent response latency of this nature could easily lead to a history of overeating and thus provide a neurobiological explanation for obesity.

\section{Reward and Hunger}

While this previous work lays the groundwork for a bottom-up approach combining biochemical and neuropsychological methods for studying brain-body modulation of hunger, other work has adopted a more cognitive approach. Morris and Dolan examined how one's hun- 
FIGURE 1. Glucose-induced hypothalamic activity in lean and obese subjects. Spatial parametric mapping localized brain activity 7-14 minutes after glucose ingestion to the ventromedial hypothalamus.

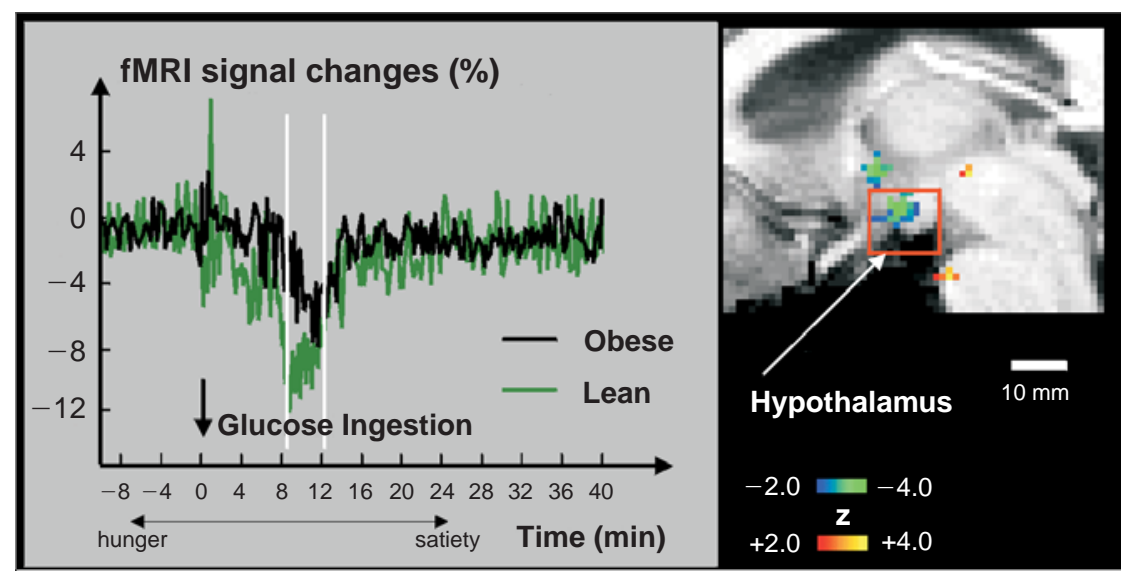

ger state can influence memory for food-related stimuli. ${ }^{37}$ Fasting subjects had increased recognition for previously viewed food stimuli over sated subjects. While the activity of limbic and limbic associated structures (hypothalamus, insula, and nucleus accumbens) covaried positively with hunger ratings and left amygdalar activity covaried positively with recognition for food items, only orbitofrontal cortex activity covaried positively as a function of food and hunger state. Specifically, the right anterior orbitofrontal cortex was found to covary positively with recognition for all stimuli (food and non-food) irregardless of hunger state, whereas the right posterior orbitofrontal cortex only covaried positively for food stimuli during the hunger (and not sated) state. This finding is intriguing in that it suggests dissociable roles for the orbitofrontal cortex and the anterior region seeking reward for correctly recognizing previously viewed stimuli, but the posterior region responsive to more internal reward. The specter of differing neural correlates for abstract versus concrete rewards arises again, but such dissociation is tenuously supported at best in the absence of corroborating data.

A shortcoming for many hunger studies is that stimuli cause both valence and arousal. Failure to control for the arousing properties of presented stimuli can confound neuroimaging studies, as increased arousal could lead to an artifactual apparent increase in reward system activity. 
To control for arousal effects, a fasting study has been performed incorporating visual stimuli from the International Affective Picture System (IAPS). ${ }^{38}$ By comparing pictures of food (and food-related) items to pictures of animals and tools matched for valence and arousal (Figure 2), the effects of these two properties on neural reward circuits can be teased apart. A comparison of food items to non-food items (low valence, low arousal) resulted in activation of the insula, the prefrontal cortex, the amygdala, the thalamus, and especially the nucleus accumbens at the ventral basal ganglia in hungry but not sated subjects. ${ }^{39}$ While these findings are indicative of brain activity specific to hunger, a comparison of food versus arousing animals (or even food-related tools to nonfood-related tools) is necessary to eliminate the confound from arousal.

\section{FUTURE DIRECTIONS}

As previously mentioned, an altered hypothalamic response to glucose ingestion may reflect the change in the central satiety signal that is associated with obesity and changes in the peripheral hormonal levels; ${ }^{9}$ however, the relationship currently lacks causal directionality. Although a study of obese individuals using the fasting paradigm described above would determine how obese individuals react differently than non-obese individuals to rewarding food stimuli while discriminating for arousal effects, such a study still cannot elucidate whether changes in reward circuitry lead to obesity or are a result of obesity. It is important to reiterate that, while animal models would be ideal for finding a double-dissociation, functional imaging on clinical populations would be more beneficial for defining the causal relationship between biochemical changes in satiety and psychobiological changes in reward values caused by obesity and substance dependence. Following are our ongoing fMRI studies in three different directions.

\section{Satiety in Prader-Willi Syndrome}

A solution for this causality issue may lie in study of patients with Prader-Willi Syndrome (PWS), a neurogenetic multisystem disorder characterized by infantile hypotonia, mental retardation, short stature, hypogonadism, dysmorphic features, and hyperphagia with a high risk of obesity. ${ }^{40}$ The behavioral symptoms in patients with PWS include compulsions toward binge eating and obesity, with typical onset just 
FIGURE 2. Arousing and non-arousing rewarding stimuli. A comparison of stimuli matched for valence and arousal (top row) allows for the identification of changes in reward processing with hunger. In contrast, a comparison of stimuli matched for valence but not arousal (columns) allows for the identification of neural changes due to arousal both independent (right column; animal) and dependent (left column; food) upon hunger state. The functional activation map at an axial brain section through the accumbens nucleus and the posterior orbitofrontal cortex (the Talairach coordinate $\mathrm{z}=-10 \mathrm{~mm}$ ) demonstrated the brain activity changes induced by food pictures during a hunger state.

Food (or food-related)
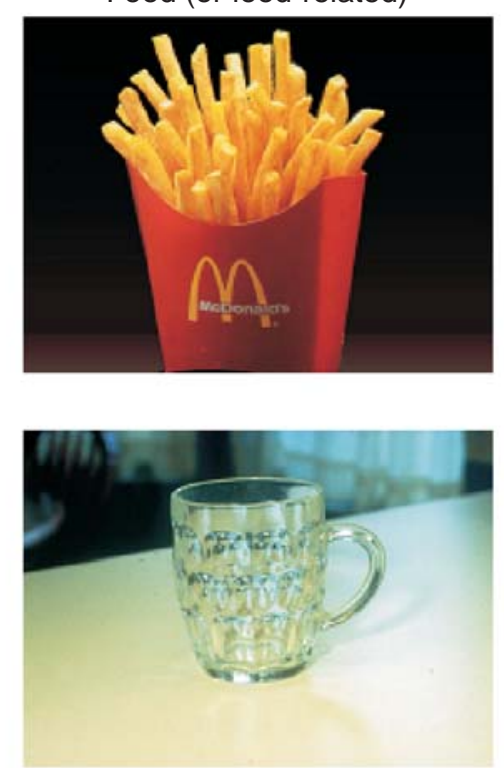

Animal
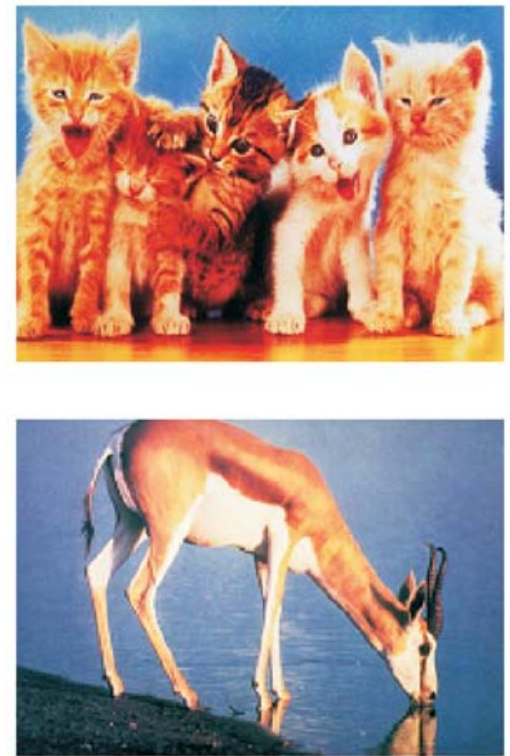

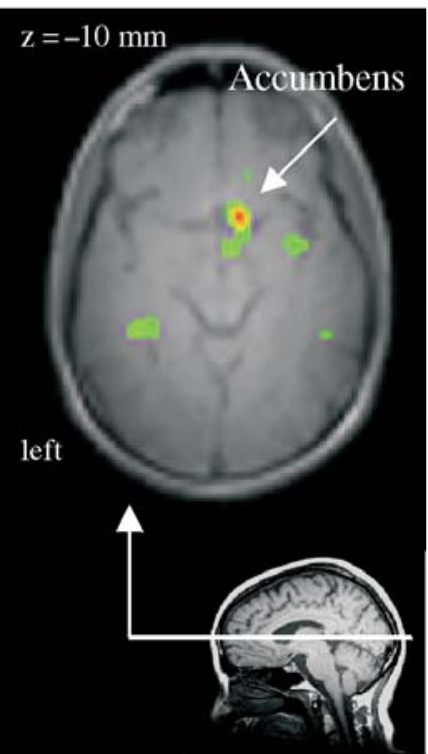


prior to puberty. An analysis of reward circuit activity using fMRI immediately following the onset of behavioral symptoms would clarify if hunger mediates reward response or vice versa. For example, a disruption of hypothalamic activity would suggest that an altered satiety response $^{41}$ eventually results in modulation of reward processing, whereas variations in reward circuit activity suggest a cognitive alteration in hypothalamic function. The running question of whether or not different reward circuits mediate abstract versus concrete rewards may well be answered by this study, as food rewards may be perceived with greater value in binge eaters.

\section{Expression of Hunger or Fasting in Hypoglycemia Unawareness}

Hypoglycemia unawareness (i.e., development of neuroglycopenia without appropriate prior awareness of autonomic warning symptoms) is a frequent and potentially dangerous syndrome and becomes the most important acute complication in type I diabetes after long-term intensive insulin treatment. Because hypoglycemia often occurs suddenly and unpredictably, patients with hypoglycemia unawareness cannot correct impending hypoglycemia (e.g., by eating food). ${ }^{42}$ Therefore, the condition of hypoglycemia unawareness can be used to address the means by which healthy individuals are consciously aware of hunger and satiety.

Although hunger and fasting are terms typically used interchangeably, the distinction needs to be made that fasting is a quantifiable biological phenomenon whereas hunger is more akin to a mood or emotional state. While hunger is an emergent state arising from fasting, cognitive processes can undoubtedly influence one's perception of hunger, resulting in marked individual differences in hunger sensation. An analysis of hypoglycemia unawareness using our current fMRI paradigms can shed light upon how the hunger state develops from fasting and what emotional or reward processing factors attenuate that state. While the interactions between satiety, cognition, and reward processing are undoubtedly complex, careful investigation of these phenomena will certainly clarify the mechanisms by which these seemingly disparate processes interact.

\section{Brain Modulation of Reward in Obese Tobacco Users}

Finally, if hunger is viewed in the context as being an addiction, the question inevitably arises of how the neural mechanisms regulating satiety and those regulating addictive rewards interact. For example, the 
nicotine literature is ripe with studies citing weight gain among chronic smokers upon smoking cessation, with this potential weight gain acting as a deterrent (especially among women) against quitting. 43,44 The finding of elevated leptin levels among chronic smokers could link hunger to addiction. ${ }^{45,46} \mathrm{~A}$ functional neuroimaging investigation into the neuroendocrine regulation of leptin among chronic smokers would have a two-fold purpose: not only would such a study further specify the mechanisms by which leptin may mediate satiety, but the potential of leptin for mediating signaling among reward circuitry would be assessed. Research into the direct interaction of hunger and reward circuitry is essential for pinning down the transitional boundaries between biology and psychology, with investigations of this nature shedding light upon the brain modulation of reward by emotion and obesity.

\section{CONCLUSIONS}

Redefining obesity as the product of a substance dependence disorder would alleviate the stigma associated with this illness while providing new directions for treating this growing epidemic. We acknowledge that the causal relationship between eating behaviors and the observed alterations in neurobiological reward circuitry remains to be established, most likely though the study of genetic conditions with accompanying eating disorders (i.e., Prader-Willi Syndrome). Although the DSM-IV does not recognize food as a substance of abuse, the converging neuroimaging, cognitive, and behavioral findings presented above suggest that food feasibly fits within the model of substance dependence. Such a framework would necessitate further study of biochemical messengers such as leptin and ghrelin, whose traditional roles in mediating hunger states may well transcend into modulating the relative reward of nonfood stimuli. Further research into these directions will grant us a truly comprehensive understanding of the complex neural and endocrine interactions driving our eating behaviors.

\section{REFERENCES}

1. Lyznicki JM, Young DC, Riggs JA, Davis RM. For the Council on Scientific Affairs. Obesity: Assessment and management in primary care. Am Fam Physician. $2001 ; 63: 2185-96$.

2. U.S. Department of Health and Human Services, Public Health Service. The Surgeon General's call to action to prevent and decrease overweight and obesity. Office of the Surgeon General. 2001; Available from: U.S. GPO, Washington. 
3. Raman RP. Obesity and health risks. J Am Coll Nutr. 2002; 21:134S-9S.

4. Pi-Sunyer FX. The medical risks of obesity. Obesity Surgery. 2002; 12(1): $6 \mathrm{~S}-11 \mathrm{~S}$.

5. Sturm R, Wells KB. Does obesity contribute as much to morbidity as poverty or smoking? Public Health. 2001; 115(3):229-35.

6. Jonas JM, Gold MS. Cocaine abuse and eating disorders. Lancet. 1986; 1:390-1.

7. Gold MS, Johnson CR, Stennie K. Eating Disorders. In: Lowinson JH, Ruiz P, Millman RB, Langrod JG, eds. Substance Abuse: A Comprehensive Textbook. 3rd ed. Philadelphia, PA: Lippincott, Williams and Wilkins, 1997:319-30.

8. Gautier JF, Chen K, Salbe AD, et al. Differential brain responses to satiation in obese and lean men. Diabetes. 2000; 49:838-46.

9. James GA, Guo W, Liu Y. Imaging in vivo brain-hormone interaction in the control of eating and obesity. Diabetes Technol Ther. 2001; 3:617-22.

10. Schwartz MW, Woods SC, Porte D Jr, et al. Central nervous system control of food intake. Nature. 2000; 404:661-71.

11. Woods SC, Seeley RJ. Understanding the physiology of obesity: Review of recent developments in obesity research. Int J Obes Relat Metab Disord. 2002; Suppl 4:S8-S10.

12. Shizgal P, Fulton S, Woodside B. Brain reward circuitry and the regulation of energy balance. Int J Obes Relat Metab Disord. 2001; 25: Suppl 5, S17-S21.

13. Saper CB, Chou TC, Elmquist JK. The need to feed: Homeostatic and hedonic control of eating. Neuron. 2002; 36:199-211.

14. Kadden RM. Behavioral and cognitive-behavioral treatments for alcoholism: Research opportunities. Addictive Behaviors. 2001; 26(4):489-507.

15. Liu Y, Gao JH, Liu HL, Fox PT. The temporal response of the brain after eating revealed by functional MRI. Nature. 2000; 405:1058-62.

16. Matsuda M, Liu Y, Mahankali S, et al. Altered hypothalamic response to oral glucose intake in obese humans. Diabetes. 1999; 48:1801-6.

17. Schultz W. Multiple reward signals in the brain. Nature Rev Neurosci. 2000; 1(3):199-207.

18. Schultz W. Getting formal with dopamine and reward. Neuron. 2002; 36: 241-63.

19. Tzschentke TM. Pharmacology and behavioral pharmacology of the mesocortical dopamine system. Prog Neurobio. 2001; 63:241-320.

20. Breiter HC, Rosen BR. Functional magnetic resonance imaging of brain reward circuitry in the human. Ann N Y Acad Sci. 1999; 877:523-47.

21. Rolls ET. The orbitofrontal cortex and reward. Cerebral Cortex. 2000; 10: 284-94.

22. Baxter MG, Murray EA. The Amygdala and Reward. Nature Rev Neurosci. 2002; 3:563-73.

23. Denton D, Shade R, Zamarippa F, et al. Neuroimaging of genesis and satiation of thirst and an interoceptor-driven theory of origins of primary consciousness. Proc Natl Acad Sci USA. 1999; 96:5304-9.

24. Augustine JR. Circuitry and functional aspects of the insular lobe in primates including humans. Brain Res Rev. 1996; 22:229-44.

25. O'Doherty J, Kringelbach ML, Rolls ET, et al. Abstract reward and punishment representations in the human orbitofrontal cortex. Nature Neurosci. 2001;4:95-102. 
26. Tataranni PA, Gautier JF, Chen K, et al. Neuroanatomical correlates of hunger and satiation in humans using positron emission tomography. Proc Natl Acad Sci USA. 1999; 96:4569-74.

27. Caggiula AR, Donny EC, White AR, et al. Cue dependency of nicotine self-administration and smoking. Pharmacology, Biochemistry and Behavior. 2001; 70:515-30.

28. Schroeder BE, Binzak JM, Kelly AE. A common profile of prefrontal cortical activation following exposure to nicotine- or chocolate-associated contextual cues. Neuroscience. 2001; 105:535-45.

29. Goldstein RZ, Volkow ND. Drug addiction and its underlying neurobiological basis: neuroimaging evidence for the involvement of the frontal cortex. Am J Psychiatry. 2002; 129:1642-52.

30. Bonson KR, Grant SJ, Contoreggi CS, et al. Neural systems and cue-induced cocaine craving. Neuropsychopharmacology. 2002; 26:376-86.

31. Lang PJ, Bradley MM, Fitzsimmons JR, et al. Emotional arousal and activation of the visual cortex: An fMRI analysis. Psychophysiology. 1998; 35:199-210.

32. Due DL, Huettel SA, Hall WG, Rubin DC. Activation in mesolimbic and visuospatial neural circuits elicited by smoking cues: Evidence from functional magnetic resonance imaging. Am J Psychiatry. 2002; 159:954-60.

33. Elliot R, Friston KJ, Dolan RJ. Dissociable neural responses in human reward systems. J Neurosci. 2000; 20:6159-65.

34. Martin-Sölch C, Magyar S, Künig G, et al. Changes in brain activation associated with reward processing in smokers and nonsmokers: A positron emission tomography study. Exp Brain Res. 2001; 139:278-86.

35. Stein DJ, Liu Y, Shapira NA, Goodman WK. The psychobiology of obsessive-compulsive disorder: How important is the role of disgust? Curr Psychiatry Rep. 2001; 3:281-7.

36. Shapira NA, Liu Y, He AG. Brain activation by disgust-inducing pictures in obsessive-compulsive disorder. Bio Psychiatry. 2003; 54:751-6.

37. Morris JS, Dolan RJ. Involvement of human amygdala and orbitofrontal cortex in hunger-enhanced memory for food stimuli. J Neurosci. 2001; 21:5304-10.

38. Lang PJ, Bradley MM, Cuthbert BN. International affective picture system (IAPS): Instruction Manual and Affective Ratings. Technical Report A-5. 2001; The Center for Research in Psychophysiology, University of Florida. Gainesville, FL.

39. James GA, He AG, Miller AW, et al. MRI of hunger and insula activation during a fasting paradigm. Proceedings of International Society for Magnetic Resonance in Medicine. 2002; 10:817.

40. Clarke DJ, Boer H, Whittington $\mathrm{H}$, et al. Prader-Willi syndrome, compulsive and ritualistic behaviors: The first population-based survey. Br J Psychiatry. 2002; 180:358-62.

41. Shapira NA, Lessig MC, Liu Y, et al. Neuroanatomical correlates of satiation and hunger in adults with Prader-Willi Syndrome: A study using fMRI. Abstract for the 17th Annual PWSA Scientific Conference 2002, Salt Lake City, CO.

42. Bolli GB. Prevention and treatment of hypoglycaemia unawareness in type 1 diabetes mellitus. Acta Diabetol. 1998; 35(4):183-93.

43. Pomerleau CS, Zucker AN, Stewart AJ. Characterizing concerns about postcessation weight gain: Results from a national survey of women smokers. Nicotine Tob Res. 2001; 3:51-60. 
44. Kennedy DT, Giles JT, Chang ZG, et al. Results of a smoking cessation clinic in community pharmacy practice. J Am Pharm Assoc (Wash). 2002; 42(1):51-6.

45. Oeser A, Goffaux J, Snead W, Carlson MG. Plasma leptin concentrations and lipid profiles during nicotine abstinence. Am J Med Sci. 1999; 318:152-7.

46. Perkins KA, Fonte C. Effects of smoking status and smoking cessation on leptin levels. Nicotine Tob Res. 2002; 4:459-66. 